\title{
Experimental Parameter Optimization for Adaptive LoRa Modulation in Body-Centric Applications
}

\author{
Thomas Ameloot, Patrick Van Torre and Hendrik Rogier \\ Department of Information Technology (INTEC) \\ Ghent University/imec, Belgium \\ thomas.ameloot@ugent.be
}

\begin{abstract}
The relentless expansion of the Internet of Things is fueled by constant innovations in low-power wide-area network technologies. Industry forerunners such as LoRa, SigFox and NBIoT continuously seek to achieve larger communication ranges while consuming as little energy as possible. These efforts, in turn, facilitate vast performance increases in a wide range of related application areas, such as body-centric communication. For example, recently, LoRa modules have been integrated onto wearable textile antennas, thereby greatly extending the range of the body-centric networks. However, as the resulting communication links need to accommodate mobile users, many nodes will regularly be communicating using suboptimal LoRa modulation parameters as these users move around. Adaptive LoRa modulation aims to solve this by optimizing these parameters in realtime, based on the location of the user and the actual performance of the wireless link. In this contribution, the optimal settings for one of the key LoRa modulation parameters, the spreading factor, are experimentally determined. More specifically, it is shown that only a very limited number of spreading factor options should be used in an adaptive LoRa modulation scheme. The results also yield insight into more general LoRa communication aspects by analyzing packet reception and channel throughput data gathered in an urban environment.
\end{abstract}

Index Terms-Body-centric communication, Outdoor Propagation, LoRa, Substrate Integrated Waveguide, Textile Antenna

\section{INTRODUCTION}

In recent years, sub-GHz, low-power communication technologies such as LoRa [1], SigFox [2] and NB-IoT [3] have seen a strong rise in popularity. These technologies facilitate the deployment of large sensor networks with nodes that can operate autonomously for years on end before running out of battery power. Additionally, by using sub-GHz frequencies, they can cover much larger areas than more traditional wireless network technologies such as WiFi (IEEE 802.11) and Bluetooth, when a lower data rate is acceptable. In addition to being one of the strongest contenders for market share, LoRa is very popular for research applications as well since it has been found to offer great performance [4]-[8], while also being license-free, allowing users to build their own networks on the link layer without having to pay for a subscription. For these reasons, but also by virtue of its slightly larger global coverage, its network layer counterpart LoRaWAN is actively being adopted for a wide range of Internet of Things (IoT) applications.

One application area where sensor network technologies such as LoRa are significantly changing the playing field is body-centric communication. As a result of the very low power usage associated with LoRa, this technology can be integrated onto wearable nodes without requiring an excessively large battery to power the on-board communication hardware. In previous research, LoRa has shown good promise for bodyto-body and body-to-base-station sensor communication applications that do not require a high data rate [9], [10]. One of the greatest strengths of this modulation technology lies without doubt in its reconfigurability. By changing the spreading factor (SF), bandwidth (BW) and code rate (CR) of a LoRa message, the communication protocol can be configured to favor either a larger range or a higher data rate. In body-centric LoRa propagation research, gathering as much measurement points as possible is usually more important than achieving a large communication range. Consequently, lower spreading factors are favored for these applications. However, this results in a systematic underestimation of the performance that can be achieved with LoRa. To explore which data rates can really be obtained at which communication ranges, a system should be implemented that actively optimises the LoRa modulation settings used at a given node location, based on a real-time assessment of the link performance. By judiciously choosing the right settings, such an optimization scheme also optimizes the data rate, which is very valuable for mobile sensor communication systems that benefit from a (relatively) higher data rate.

Some more details and considerations concerning this proposed system are discussed in the next section. Then, in Section III, the measurement strategy and setup used to determine some of the key design considerations and performance predictions for such a system are introduced. The data gathered using this setup are presented and analyzed in Section IV and finally, in Section V, a conclusion to this work is formulated.

\section{Proposed System}

As previously mentioned, the premise of this work is to get both sides of a body-to-base-station LoRa link to agree on a set of modulation settings that co-optimize the data rate at which they communicate and the coverage of the network they are in. As seen in Table I, the LoRa receiver sensitivity specifications provided by Semtech for its most popular SX1276 LoRa radio [11] show that the largest variation in link performance results from varying the LoRa spreading factor (SF). Due to the popularity of using a bandwidth (BW) of $125 \mathrm{kHz}$ and the added complexity of optimizing both bandwidth and spreading 
factor, a fixed BW setting of $125 \mathrm{kHz}$ will be used for all of the experiments in this contribution. For similar reasons, a fixed code rate (CR) of $4 / 5$ is selected. Additionally, it should be noted that when already using the largest spreading factor and smallest bandwidth, one last way of increasing the data rate is switching to a different modulation technology supported by the LoRa radio such as frequency shift keying (FSK) However, as this work is in the scope of characterizing the performance of LoRa links, this option will not be considered further.

TABLE I: $868 \mathrm{MHz}$ LoRa receiver sensitivities (in $\mathrm{dBm}$ ) for different bandwidth (BW) and spreading factor (SF) settings.

[11]

\begin{tabular}{|c|c|c|c|c|c|c|c|}
\hline & \multicolumn{6}{|c|}{ SF } \\
\hline & & 7 & 8 & 9 & 10 & 11 & 12 \\
\hline \multirow{3}{*}{ BW } & $125 \mathrm{kHz}$ & -123 & -126 & -129 & -132 & -133 & -136 \\
\hline & $250 \mathrm{kHz}$ & -120 & -123 & -125 & -128 & -130 & -133 \\
\hline & $500 \mathrm{kHz}$ & -116 & -119 & -122 & -125 & -128 & -130 \\
\hline
\end{tabular}

Since LoRa transmits in public ISM-bands, a duty cycle restriction between 1 and $10 \%$ is imposed by law, depending on the specific ISM-band. This work explores the performance of LoRa in the $868 \mathrm{MHz}$ band, where the strictest rules apply. This severe limitation on the time that a communication system is allowed to use the band should be taken into account when implementing an adaptive LoRa modulation scheme.

The amount of air time required to transmit a LoRa packet is inversely proportional to the useful bit rate $R_{b}$ of a LoRa link, which can be calculated using [11]

$$
R_{b}=S F \cdot \frac{B W}{2^{S F}} \cdot C R .
$$

However, as can be seen in Fig. 1, lab measurements performed with a radio frequency signal analyzer and a commercial LoRa transceiver show that some packet sizes are more efficient than others, which is due to how information is encoded in a LoRa message. In Fig. 1, the air time needed to transmit a packet of a certain length with a certain spreading factor is indeed described by a stepped line instead of the smooth line that would be expected from 1 .

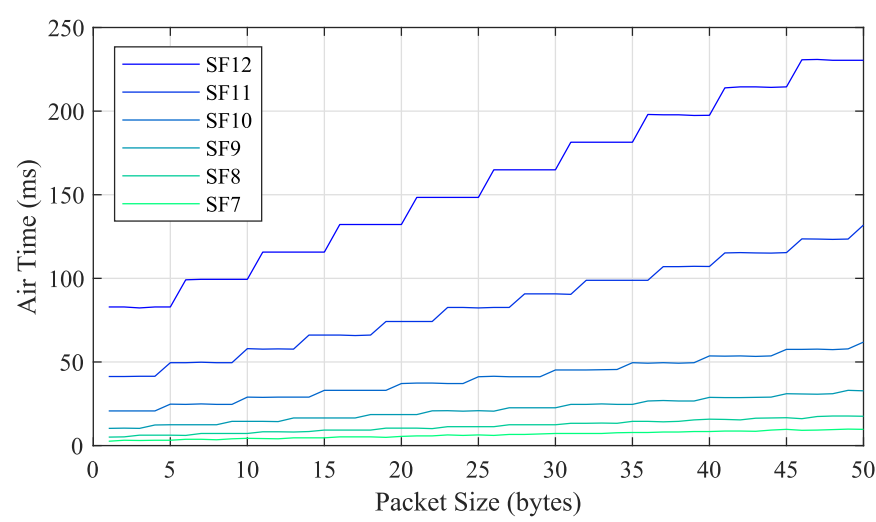

Fig. 1: Experimentally determined air time for packets transmitted with different spreading factors (SF) and packet sizes.
Based on these air times, the maximum data rates that can be achieved for each of the spreading factor and packet size choices shown in Fig. 1 were evaluated. The results are shown in Fig. 2.

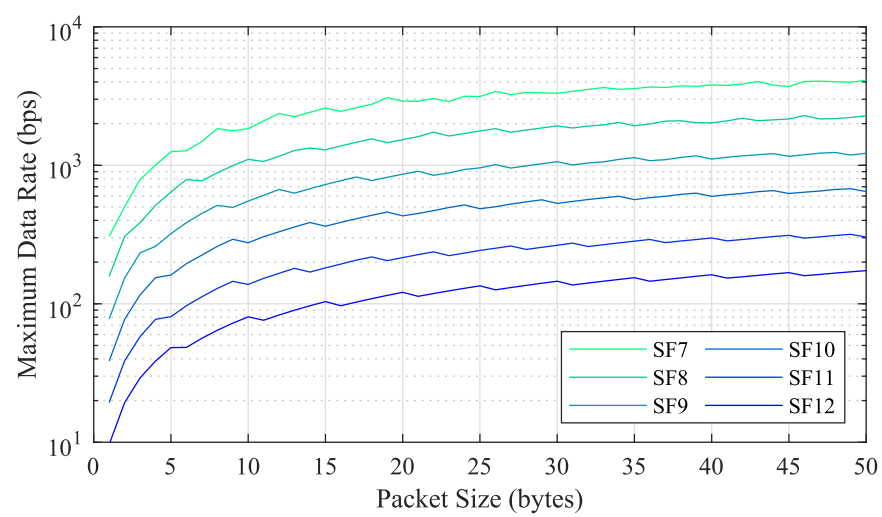

Fig. 2: Maximum data rates for packets transmitted with different spreading factors (SF) and packet sizes, based on the air times presented in Fig. 1.

From Fig. 2, one might decide that the packet size should be chosen as large as possible to optimize the data rate. However, the packet loss probability should also be taken into account. For example, when choosing a large message payload size to reduce the impact of communication overhead, a lost packet may have a very severe impact on the achieved data rate. As the channel between mobile wireless nodes can be heavily impacted by packet loss, practical link characterization data should be used to determine the optimal packet size. Additionally, for all current LoRa transceivers, using larger packet sizes also limits the rate at which the channel can be probed, limiting the amount of data available to perform accurate channel estimation.

Another hurdle to realize adaptive LoRa modulation lies in constructing a good algorithm to determine how and when to switch between certain modulation settings. Naturally, messages sent with different settings than those expected by the receiver will not be received and, as such, there needs to be a robust way for nodes to commonly decide on which settings to use for the next packet. Reliably agreeing on these settings could prove to be a real challenge, especially when having to comply with the duty cycle limitations currently imposed on the relevant ISM-bands.

\section{MEAsurement SETUP}

An exploratory measurement campaign was performed to make informed choices about how to configure a wearable LoRa communication system with a dynamic data rate and coverage co-optimization scheme and how to accurately predict the performance of such a system. Therefore, a LoRa link between a base-station and a test person equipped with wearable LoRa nodes was characterized. Along with descriptions of the measurement methodology and the propagation environment used for this test, a brief overview of the wearable hardware is given in the next subsections. 


\section{A. Hardware}

The wireless nodes applied to characterize the body-centric LoRa link are based on the custom-built research-oriented LoRa platform described in [12]. One of these nodes serves as a base-station. Therefore, it is connected to a vertically polarized omnidirectional antenna. This base-station was placed on the roof of a modern office building, at a height of $57 \mathrm{~m}$.

On the mobile side of the link, a test person was outfitted with two wearable LoRa nodes. To construct these nodes, the LoRa platform mentioned earlier was integrated onto a substrate integrated waveguide (SIW) antenna implemented in bendable textile materials. This yields a low-profile system that can be worn unobtrusively on the front and back of the torso. The test person was equipped with two nodes because the radiation patterns of each textile antenna only reliably covers a single hemisphere. Combining the results from two nodes worn on opposite sides of the body also mitigates the signal losses caused by body shadowing.

\section{B. Measurement Strategy}

The LoRa base-station discussed in the previous subsection was configured to continuously transmit very short packets while constantly varying the spreading factor of the LoRa modulation. All packets are sent with a transmit power of $10 \mathrm{dBm}$. The receive nodes are synchronized with the transmitter through their internal clocks. They update their clocks every time a packet is received with a spreading factor of 12 , since these packets are the most likely to be received. Upon each packet reception, the receiving nodes also measure the signal-to-noise ratio (SNR) of the LoRa message and store this value in their flash memory, along with a timestamp and packet number. This enables us to relate this value to the location of the test person, which is logged using a GPS app on his smartphone.

\section{Propagation Environment}

As mentioned earlier, the base-station (TX) was placed at a height of $57 \mathrm{~m}$, on the roof of a modern office building. As seen in Figs. 3 and 4, the test person moved along a number of trajectories to the north of this building. Most notably, he traveled along both sides of the nearby canal and along the wide avenue running from the oddly shaped roundabout (marked with $\mathrm{a} *$ ) in the north of the area to the high-tech industry park south of this canal. It is important to note that this avenue crosses the waterway and a nearby highway by means of a relatively wide bridge, providing a terrain that is slightly elevated compared to the surrounding area, whereas the roads next to the canal are slightly more receded than most of the other terrain.

\section{Measurement Results and Analysis}

To assess the performance of the LoRa link setup described in the previous section, the SNR values were registered for all successfully received packets. The results are shown in Fig. 3. First, this figure shows that there are a lot of areas where the link is shadowed by obstacles in the environment. For example, the (already slightly receded) road on the south side of the canal is almost completely shadowed by buildings and trees. Additionally, for a lot of packets that were received, a negative SNR was registered, which again shows the benefits of using a modulation technology such as LoRa for outdoor off-body applications. Furthermore, it is also apparent that certain areas very close to the base-station are less well covered due to shadowing by the building. Yet, it should also be mentioned that, in contrast to the link performance in these non-lineof-sight (NLoS) areas, packet reception was a lot higher in line-of-sight $(\mathrm{LoS})$ areas, such as along the avenue crossing the canal.

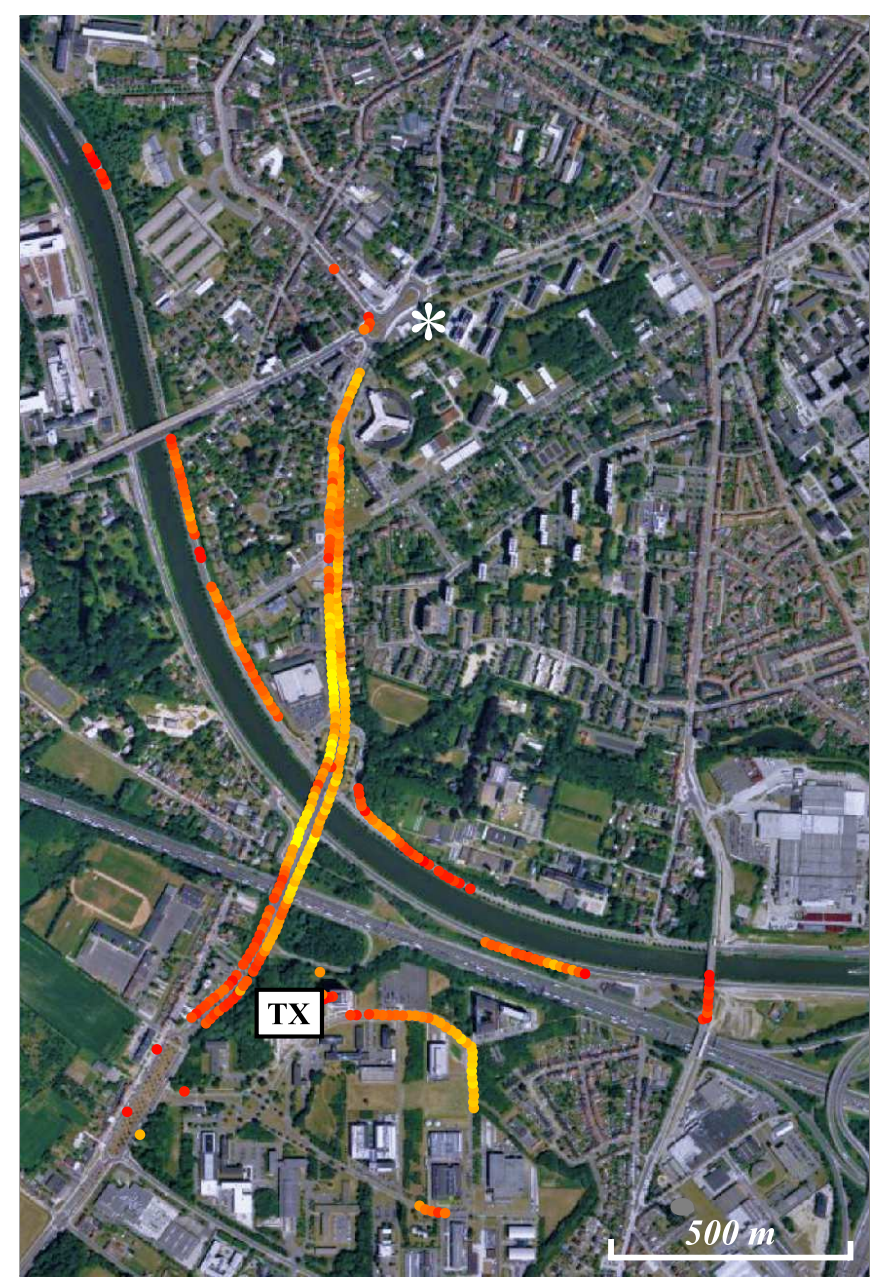

Signal-to-Noise Ratio

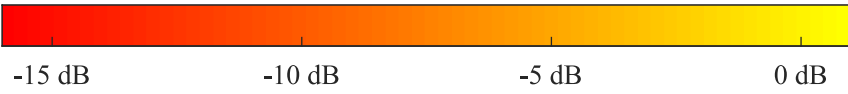

Fig. 3: Signal-to-noise ratios measured by the wearable LoRa nodes after applying selection combining.

While the SNR is a good measure for judging the strength and reliability of these communication links, it does not really provide a lot of information on how LoRa modulation can be adapted to suit the needs of a mobile user. Therefore, Fig. 4 shows the lowest spreading factor that enabled the receivers to demodulate packets along the test trajectories. When comparing the coverage that is achieved when using 
a spreading factor of 12 to the coverage achieved for $\mathrm{SF}=7$, a notable increase is seen. This implies that, depending on the speed of the mobile user and the density of scatterers in the environment, there indeed are a good number of areas where dynamically adjusting the spreading factor can yield noticeable improvements to the performance of the communication links.

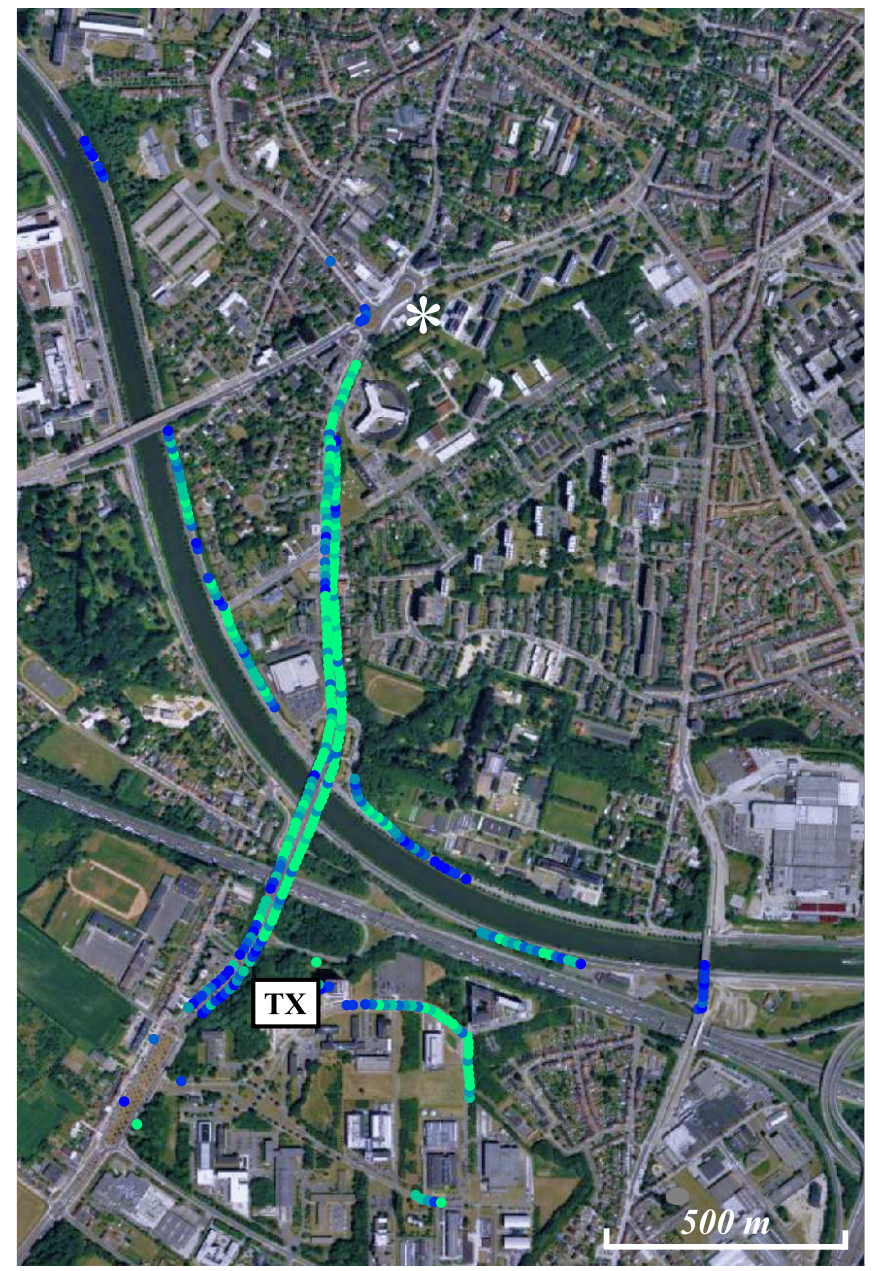

LoRa Spreading Factor

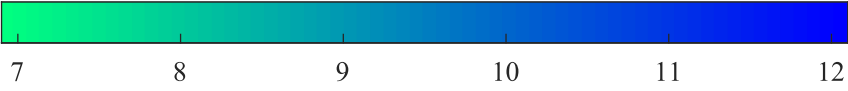

Fig. 4: Lowest spreading factor settings that result in successful packet reception.

Yet, identifying the best way to design a spreading factor optimization scheme requires a deeper analysis of the data presented above. As such, looking at the cumulative packet reception ratios (PRRs) for increasing SNR values, as shown in Fig. 5, yields a lot of insight in the performance of different SF settings. This characteristic shows how the use of higher spreading factors is beneficial to the packet reception ratio for all received power levels. It also shows how many packets will still be received for a given spreading factor when for example only those areas with the lowest SNRs are considered.

Ultimately, multiplying the data in Fig. 5 with the data rates, as presented in Fig. 2, yields the cumulative throughput of the LoRa channel for each of the SF settings (Fig. 6). In turn,

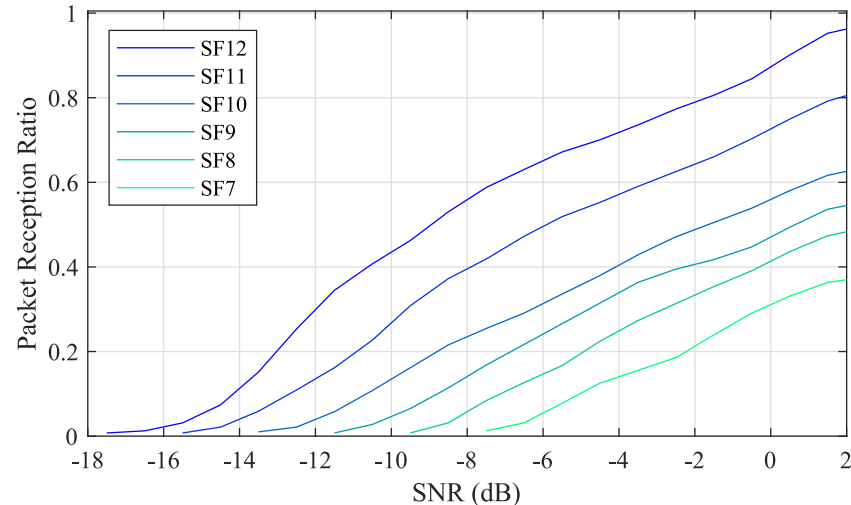

Fig. 5: Cumulative packet reception ratios for rising SNR values in all areas that have coverage when $\mathrm{SF}=12$.

these data are easily transformed into the effective throughput shown in Fig. 7. This characteristic shows which spreading factor results in the highest data rate. Therefore, the plot is a good reference for selecting the optimal spreading factor for different levels of SNR. To this end, a decision table was constructed (Table II), which also includes the maximum data rate, as shown in Fig. 2 for three different packet sizes.

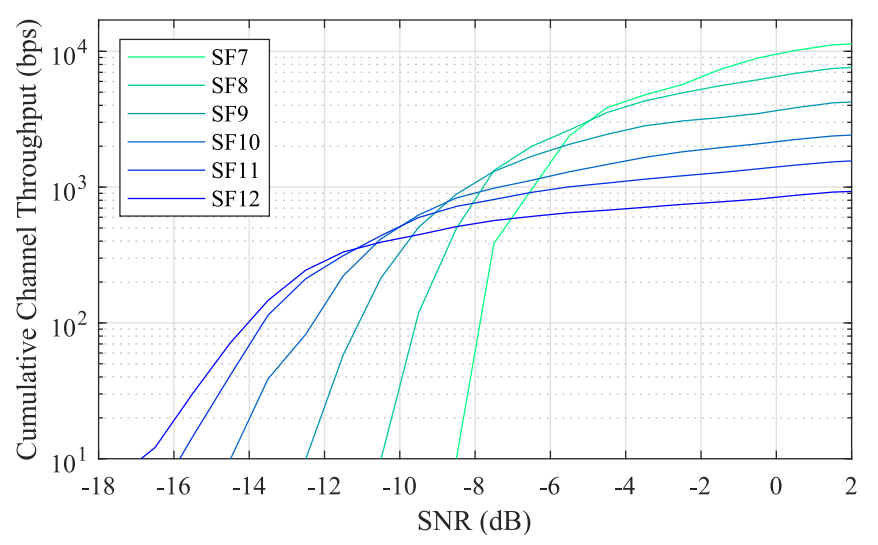

Fig. 6: Cumulative channel throughput as a fuction of the SNR (for continuous bursts with packet size $=1$ byte).

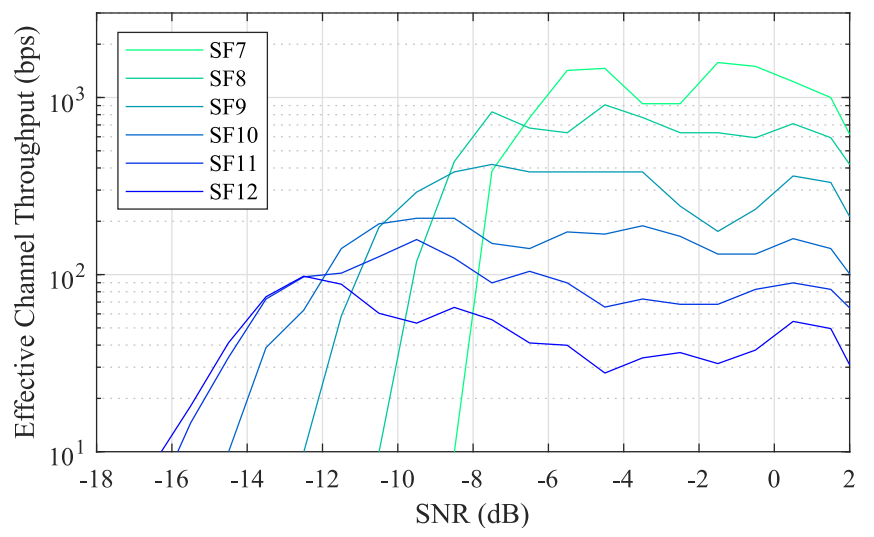

Fig. 7: Effective channel throughput as a fuction of the SNR (for continuous bursts with packet size $=1$ byte). 
TABLE II: Optimal spreading factor for a given SNR range and the corresponding maximum data rate, when transmitting with packet sizes of 1,10 and 50 bytes.

\begin{tabular}{|c|c|c|c|c|}
\hline \multirow{2}{*}{ SNR Range } & \multirow{2}{*}{ Optimal SF } & \multicolumn{3}{|c|}{ Maximum data rate (bps) } \\
\cline { 3 - 5 } & & 1 byte & 10 bytes & 50 bytes \\
\hline$]-7, \ldots$ & 7 & 307 & 1840 & 4119 \\
{$[-9,-7]$} & 8 & 158 & 1104 & 2281 \\
{$[-11,-9]$} & 9 & 78 & 552 & 1221 \\
{$[-12,-11]$} & 10 & 39 & 276 & 646 \\
{$[-13,-12]$} & 11 & 19 & 138 & 303 \\
$\ldots,-13]$ & 12 & 10 & 80 & 174 \\
\hline
\end{tabular}

Of course, when designing a spreading factor switching protocol based on these metrics, some other application parameters should be considered as well. Given the relatively small differences in performance between neighbouring spreading factors, an adaptive optimization algorithm that switches between all possible spreading factors will probably be quite inefficient. Instead, switching between two or three preset levels may significantly reduce the overhead of switching while retaining the benefits offered by adapting the spreading factor to the propagation conditions. Naturally, the most obvious choices for a system that switches between two spreading factors are SF 7 and SF 12. A possible third option might be SF 9 or SF 10, depending on the specific propagation environment.

Another factor that should be taken into account is the speed of the mobile user. Based on Fig. 4, it is clear that for a fast-moving receiver (such as a cyclist), the time spent in zones where only packets sent with a high spreading factor are received is limited to only a few seconds. This means that the added value of switching the spreading factor during those short moments is fairly low, which even advocates against using any form of adaptive SF algorithm. In contrast, for lower (walking) speeds, the time spent in zones where only those packets with a high spreading factor are received might be a lot longer, which does justify the use of adaptive LoRa modulation.

Additionally, it should be mentioned that the limited duty cycle that is imposed on LoRa by law could be a serious hurdle for probing the channel and deciding when to change the spreading factor. Yet, for a system that only uses two or three SF options, as proposed earlier, this might not be a serious issue, since the lower amount of SF choices greatly simplifies this decision process.

A final option consists of changing the spreading factor based on positioning data and thus decide on changing certain modulation settings based on prior knowledge about the propagation environment. Ideally, in this kind of scheme, the wireless communication should be set up when channel conditions are favorable while the system should be inactive when the connection is likely to degrade.

\section{CONCLUSION}

Within the research framework that proposes an adaptive LoRa modulation system for use in body-centric communication networks, this contribution empirically determines how the spreading factor (SF) influences the performance of a mobile body-to-base-station LoRa link. Optimal SF switching parameters were determined (Table II) based on link performance data gathered in an outdoor measurement campaign, yielding insight into how a mobile LoRa system may adapt its modulation settings to optimize the data rate while maintaining the largest possible coverage area. It was concluded that, due to the small differences in performance between neighbouring spreading factors, adaptive LoRa modulation will only be useful when implemented with a very small number of SF options. Future work includes determining a good SF switching algorithm and communication protocol to decide when to switch between different LoRa settings and to avoid losing the connection, while respecting the duty cycle limit of $1 \%$ imposed on $868 \mathrm{MHz}$ LoRa links. Despite this obvious challenge, adaptive LoRa modulation is still regarded as a viable way to improve the performance of communication links in applications that benefit from increased data rates on the one hand, but still rely on achieving the best possible coverage on the other hand.

\section{ACKNOWLEDGMENT}

This work was partly funded by the Research Foundation Flanders (FWO) through the "MUlti-SErvice WIreless NETwork", FWO-FRS Excellence of Science - EOS project.

\section{REFERENCES}

[1] LoRa Alliance. [Online]. Available: https://www.lora-alliance.org/

[2] Sigfox. [Online]. Available: https://www.sigfox.com/en

[3] "NarrowBand - Internet of Things (NB-IoT)," GSM Association. [Online]. Available: https://www.gsma.com/iot/narrow-band-internet-ofthings-nb-iot/

[4] U. Noreen, A. Bounceur, and L. Clavier, "A study of LoRa low power and wide area network technology," in 2017 International Conference on Advanced Technologies for Signal and Image Processing (ATSIP), May 2017, pp. 1-6.

[5] J. Haxhibeqiri, F. Van den Abeele, I. Moerman, and J. Hoebeke, "LoRa Scalability: A Simulation Model Based on Interference Measurements," Sensors, vol. 17, no. 6, 2017. [Online]. Available: http://www.mdpi.com/1424-8220/17/6/1193

[6] A. Augustin, J. Yi, T. Clausen, and W. M. Townsley, "A Study of LoRa: Long Range \& Low Power Networks for the Internet of Things," Sensors, vol. 16, no. 9, 2016. [Online]. Available: http://www.mdpi.com/1424-8220/16/9/1466

[7] O. Georgiou and U. Raza, "Low Power Wide Area Network Analysis: Can LoRa Scale?" IEEE Wireless Communications Letters, vol. 6, no. 2 , pp. 162-165, April 2017.

[8] K. Mikhaylov, J. Petäjäjärvi, and T. Haenninen, "Analysis of Capacity and Scalability of the LoRa Low Power Wide Area Network Technology," in European Wireless 2016; 22th European Wireless Conference, May 2016, pp. 1-6.

[9] P. Van Torre, T. Ameloot, and H. Rogier, "Wearable $868 \mathrm{MHz}$ LoRa Wireless Sensor Node on a Substrate-Integrated-Waveguide Antenna Platform," in 49th European Microwave Conference, 1st - 3rd October 2019, June 2019.

[10] P. Van Torre, T. Ameloot, and H. Rogier, "Long-range body-to-body LoRa link at $868 \mathrm{MHz}$," in 2019 13th European Conference on Antennas and Propagation (EuCAP), March 2019, pp. 1-5.

[11] $137 \mathrm{MHz}$, to $1020 \mathrm{MHz}$. Low Power Long Range Transceiver, Semtech Corporation, 8 2016, rev. 5.

[12] T. Ameloot, P. Van Torre, and H. Rogier, "A Compact Low-Power LoRa IoT Sensor Node with Extended Dynamic Range for Channel Measurements," Sensors, vol. 18, no. 7, p. 2137, Jul 2018. [Online]. Available: http://dx.doi.org/10.3390/s18072137 